\title{
Spatial Correlation Based Medium Access Control Protocol Using DSR \& AODV Routing In Wireless Sensor Networks For Energy Efficient Data Collection
}

\author{
Ashutosh Kumar Shrivastava ${ }^{1}$, Dr. Rajeev Paulus ${ }^{2}$, Prof A K Jaiswal ${ }^{3}$ \\ ${ }^{I}$ (M.Tech.Student Of ECE ,SSET,SHIATS, Allahabad,India) \\ 2 (Prof Of ECE, SSET,SHIATS,Allahabad, India) \\ ${ }^{3}$ (Prof \& Head Of ECE ,SSET,SHIATS,Allahabad, India)
}

\begin{abstract}
In Wireless sensor network sensor nodes have a limited battery life and their efficient utilization is a very much importent task. Their are many ways are proposed for efficient utilization of energy. For efficient energy utilization many topologies,protocals are proposed by the help of which we can maximize the battery life. In this paper we propesed a methode in which a correlation is made between all the sensor nodes including ME(mobile element). A Vector Quantization methode are used for distance calculation between all the sensor nodes and mobile element. After finding the corrélation we used the DSR \& AODV routing Protocol. The performance of the proposed protocol has been examined and evaluated with the NS-2 simulator in terms of packet drop ratio and energy consumption. The simulation result shows that the proposed protocol with AODV routing gives a batter result compared with same protocol with DSR routing.
\end{abstract}

Keywords: ME, DVT, DSR, AODV, Wireless Sensor Network, Efficient Energy Utilization

\section{INTRODUCTION}

In wireless sensor network the main source of energy of any sensor nodes is battery which is nonrechargeable. This means that when the battery of any sensor node is discharged, it is out of service. Hence we have to utilize the available battery life very efficiently. WSN consists of a wireless communication unit and a processing unit, a sensing unit, and a power supply unit. The main aim objective is to maximize the life of power supply unit, a non-rechargeable source of energy either by improving the topology or the routing protocol

Primary source of energy to sensor node is the battery. At regular interval of time, the nodes available in a sensor network collect the data points and transform all the data points into an equivalent electric signal and distribute the signal to the sink or base node via some reliable communication medium. Sensor nodes are spatially distributed in nature and the ambient conditions related to surrounding environment of the sensors are measured by sensing circuitries in sensor network.

The MAC protocol deals with radio transmission and reception on a wireless medium which is shared. Hence an improvement on MAC protocol will give a better result hence we have used spatial correlation based MAC protocol. In this paper we used the concept that in any network topology where many number of nodes are deployed they all do not want to transmit the massage at the same time.

Hence the region where massage sending probability are high selected from all the available nodes and a mobile node will go through each node and collect the available data. According to the mobile element movement the distance between the mobile element and rest of the sensor node will change every time when the mobile element changes its position. From the calculated distance mobile element select the nearest node which wants to send the massage and set it as a sink node. The sink node is a node which collects the massage from its neighbors and stores it until the mobile element collect the data from the sink node and deliver it to the base station.

\section{RELATED WORK}

A brief literature survey is presented in the following section.

The Iterative Node Selection (INS) algorithm running at sink, determines the minimum number of representative node to complete the task based on the distortion constraint Dmax. Average distance between the sensor node is determined and informed to all the sensor nodes in the event field[1]. Based on the theoretical framework, a distributed, spatial Correlation-based Collaborative Medium Access Control (CCMAC) protocol is then designed which has two components: Event MAC (E-MAC) and Network MAC (N-MAC). E-MAC filters out the correlation in sensor records while N-MAC prioritizes the transmission of route-thru packets [2]. 
For example, intuitively, due to the spatial correlation, data from spatially separated sensors is more useful to the sink than highly correlated data from nodes in proximity. Therefore, it may not be necessary for every sensor node to transmit its data to the sink; instead, a smaller number of sensor measurements might be adequate to communicate the event features to the sink within a certain reliability/fidelity level [3].

When a specific source node " $n_{i}$ " transmits its event record to the sink, all of its correlation neighbours have redundant information with respect to the distortion constraint, $\mathrm{D}_{\max }$. This redundant information, if sent, increases the overall latency and contention within the correlation region and results in wasting scarce WSN energy resources. The proposed spatial correlation based collaborative MAC protocol aims to prevent the transmission of such redundant information and in addition, prioritize the forwarding of filtered data to the sink $[1]$.

In the paper we are going to use the DSR and AODV protocol and would work on to show that the AODV routing with spatial correlation will give a batter result compared with DSR. The DSR protocol allows nodes to dynamically discover a source route across multiple network hops to any destination in the ad hoc network. Each data packet sent then carries in its header the complete, ordered list of nodes through which the packet must pass, allowing packet routing to be trivially loop-free and avoiding the need for up-to-date routing information in the intermediate nodes through which the packet is forwarded. By including this source route in the header of each data packet, other nodes forwarding or overhearing any of these packets may also easily cache this routing information for future use [4].

AODV uses a broadcast route discovery mechanism as is also used with modifications in the Dynamic Source Routing DSR algorithm Instead of source routing however AODV relies on dynamically establishing route table entries at intermediate node. This difference pays off in networks with many nodes where a larger overhead is incurred by carrying source routes in each data packet [5].

The mobile element will calculate the position of each node and select the nearest node which want to transmit the data and in the range of mobile element. Then all the other nodes accept mobile element one node is selected and which is the closest to the mobile element are set as the sink node. The sink node will collect all the data from the other node who wants to transmit and deliver it to the mobile element. Now the mobile element will transmit all the data received from the sink node to the base station through the node which is nearest to the base station.

\section{PROPOSED MODEL}

Primary source of energy to sensor node is the battery. At regular interval of time, the nodes available in a sensor network collect the data points and transform all the data points into an equivalent electric signal and distribute the signal to the sink or base node via some reliable communication medium. Sensor nodes are spatially distributed in nature and the ambient conditions related to surrounding environment of the sensors are measured by sensing circuitries in sensor network. Proposed WSN model consisting of 11 sensor nodes deployed in a 500 × $500 \mathrm{~m}_{2}$ sensor field. Network model is simulated using Ns2 with $1 \mathrm{~mW}$ of transmitting power and $1 \mathrm{~mW}$ of receiving power, $0.001 \mathrm{mw}$ of sleep power and $1 \mathrm{~mW}$ of idle power. $2 \mathrm{MHz}$ range of bandwidth is considered along with $1 \mathrm{Mbps}$ data rate with the initial energy of 1000 joules.

\section{SPATIAL CORRELATION IN WIRELESS SENSOR NETWORK}

In wireless sensor network, when an event occurs in a sensor field, the nodes which are very nearer to that event area detect the event information and it is sensed by the neighbourhood nodes. Every node transmits its own data to sink which is highly correlated that results in redundant transmission. It is not requisite for all the nodes in the sensor field to send the data to the sink. Redundant transmission in a network is reduced by decreasing the selecting the subset of sensor nodes. Node selection technique is used to identify the representative nodes resulting in minimum energy consumption in WSN. For that, Iterative Node Selection (INS) algorithm is introduced in this paper [1].

The model for the information collected by $\mathrm{N}$ sensors in an event area is illustrated in Fig. 1. The sink is interested in estimating the event $\mathrm{S}$ in the sensor field, according to the observations of the sensor nodes, ni, in the event area [2].

Each sensor node ni observes Xi[n], the noisy version of the event information, $\mathrm{Si}[\mathrm{n}]$, which is spatially correlated to the event source, S. In order to communicate this observationto the sink through the WSN, each node has to encode its observation. The encoded information, Yi[n], is then sent to the sink through the WSN. The sink, at the other end, decodes this information to obtain the estimate, $\hat{s}$, of the event $\mathrm{S}$ from the sensor field. The encoders and the decoders are denoted by E and D in Fig. 1, respectively. 


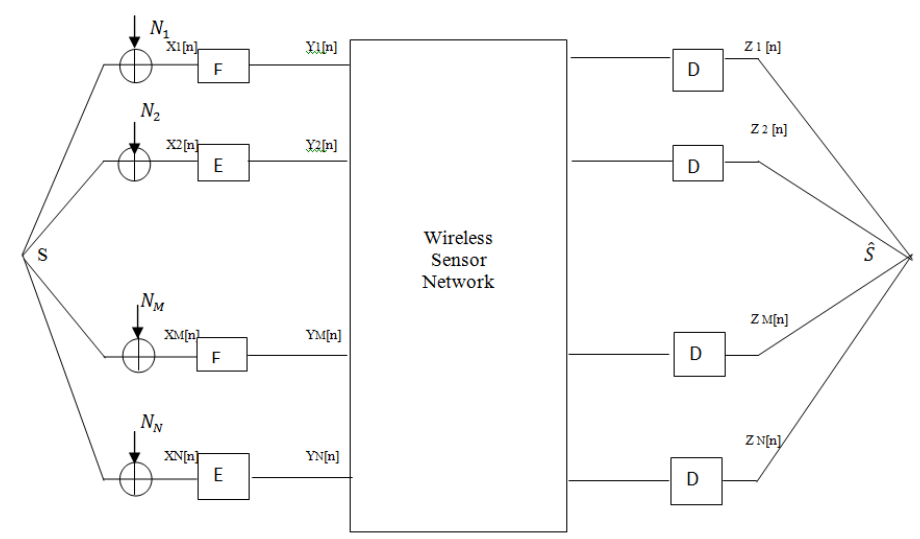

Fig1

The main objective of WSN is to estimate the event, $\mathrm{S}$, from collective observations of the sensor nodes within a reliability measure required by the application. This reliability measure is chosen as the distortion between the event source, $\mathrm{S}$, and its estimation, $\hat{s}$, at the sink. This event distortion is given by

$$
\mathrm{DE}=\mathrm{E}[\mathrm{d}(\mathrm{S}, \widehat{\mathrm{S}},)] \text {, }
$$

Where $\operatorname{dE}(\mathrm{S}, \hat{S}$,$) is the distortion measure. In this paper, we refer to the maximum distortion allowed by the$ sensor application as the distortion constraint. Our goal is to exploit spatial correlation without compromising the distortion constraint.

The event source in interest can be modelled as a random process $s(t, x, y)$ as a function of time $t$ and spatial coordinates $(x, y)$. Each observed sample, $X i[n]$, of sensor node, ni, at time $t=\operatorname{tn} 2$ is represented as

$$
\mathrm{X}_{\mathrm{i}}[\mathrm{n}]=\mathrm{Si}[\mathrm{n}]+\mathrm{N}_{\mathrm{i}}[\mathrm{n}], \mathrm{i}=1, \ldots, \mathrm{N},
$$

Where the subscript i denotes the spatial location of the sensor node ni, i.e. (xi, yi), Si[n] is the realization of the space-time process $\mathrm{s}(\mathrm{t}, \mathrm{x}, \mathrm{y})$ at $(\mathrm{t}, \mathrm{x}, \mathrm{y})=(\mathrm{tn}, \mathrm{xi}, \mathrm{yi})$, and $\mathrm{Ni}[\mathrm{n}]$ is the observation noise. $\{\mathrm{Ni}[\mathrm{n}]\} \mathrm{n}$ is a sequence of i.i.d Gaussian random variables of zero mean and variance $2 \mathrm{~N}$. We further assume that the noise each sensor node encounters is independent of each other, i.e., $\mathrm{Ni}[\mathrm{n}]$ and $\mathrm{Nj}[\mathrm{n}]$ are independent for $\mathrm{i} \neq \mathrm{j}$ and $\forall \mathrm{n}$.

Since we only consider the spatial correlation between nodes, we can assume that the samples are temporally independent. Hence, by dropping the time index n, (2) can be restated as

$$
\mathrm{X}_{\mathrm{i}}=\mathrm{Si}_{\mathrm{i}}+\mathrm{N}_{\mathrm{i}}, \mathrm{i}=1, \ldots, \mathrm{N} \text {, }
$$

Where the observation noise Ni of each sensor node ni is modelled as i.i.d. Gaussian random variable of zero mean and variance $\sigma_{N}^{2}$. The event information, $\mathrm{Si}$, at each point of the event area is modelled as joint Gaussian random variables (JGRVs) as

$$
\begin{gathered}
\mathrm{E}\{\mathrm{Si}\}=0, \operatorname{var}\{\mathrm{Si}\}=\sigma_{s}^{2} \mathrm{i}=1, \ldots, \mathrm{N},(4) \\
\rho_{(i, j)}=K_{v}\left(d_{(i, j)}\right)=\frac{\mathrm{E}\left[S_{i} S_{j}\right]}{\sigma_{s}^{2}}
\end{gathered}
$$

Where $\rho_{(i, j)}$ and $\mathrm{d}(\mathrm{i}, \mathrm{j})=\|\mathrm{si}-\mathrm{sj}\|$ denote the correlation coefficient and the distance between nodes ni and nj located at coordinates si and sj, respectively, and $\mathrm{K} \vartheta(\cdot)$ is the correlation model.

Note that, the event source, S, is also a JGRV with the same properties. Since it is of special interest, the correlation coefficient, and the distance between the event source $\mathrm{S}$ and the node ni are denoted by $\rho_{(s, i)}$ and $\mathrm{d}(\mathrm{s}, \mathrm{i})$, respectively, throughout our discussions.

The covariance function, $K \vartheta(\cdot)$, used in (4), models the relation between the correlation coefficient between the sensor observations, $\rho_{(i, j)}$, and the distance, $\mathrm{d}(\mathrm{i}, \mathrm{j})$, between the nodes ni and nj. The covariance function is assumed to be nonnegative and decrease monotonically with the distance $\mathrm{d}(\mathrm{i}, \mathrm{j})=\|\mathrm{si}-\mathrm{sj}\|$, with limiting values of 1 at $\mathrm{d}=0$ and of 0 at $\mathrm{d}=\infty$. Generally, covariance models can be classified into four standard groups as Spherical, Power Exponential, Rational Quadratic, and Mat'ern [4]. Although our results about the distortion function apply to all the covariance models, we use the Power Exponential model in this paper because the physical event information such as, electromagnetic waves, is modelled to have an exponential autocorrelation function.

Hence, the covariance function, $\mathrm{K} \vartheta(\cdot)$, is given as

$$
K_{\vartheta}^{P E}=e^{\left(-\frac{d}{\theta 1}\right) \theta 2} ; \text { for } \theta 1>0, \theta 2 \epsilon(0,2)
$$

where for $\theta 2=1$, the model becomes exponential. 


\section{A. Iterative Node Selection Algoritham}

The INS algorithm running at sink, determines the minimum number of representative node to complete the task based on the distortion constraint $D_{\max }$. Average distance between the sensor node is determined and informed to all the sensor nodes in the event field. Each node in WSN field then performs spatial correlation based collaborative MAC operation. The INS requires the statistical properties of the node distribution as input. They are density of the network, type of the network and type of node distribution. The selection of locations of correlated points based on a distortion constraint has been analyzed by means of vector quantization (VQ) algorithm.

Correlation Radius and Correlation Region are two important observations in correlation region.

* Correlation Radius - It is represented by rcorr. It is the radius of correlation region.

- Correlation Neighbour - A node $\mathrm{n}_{\mathrm{j}}$ is said to be the correlation neighbor of node $\mathrm{n}_{\mathrm{i}}$ if the distance $\mathrm{d}_{\mathrm{i}, \mathrm{j}}$ of the node $n_{i}$ is smaller than $r_{\text {corr }}$

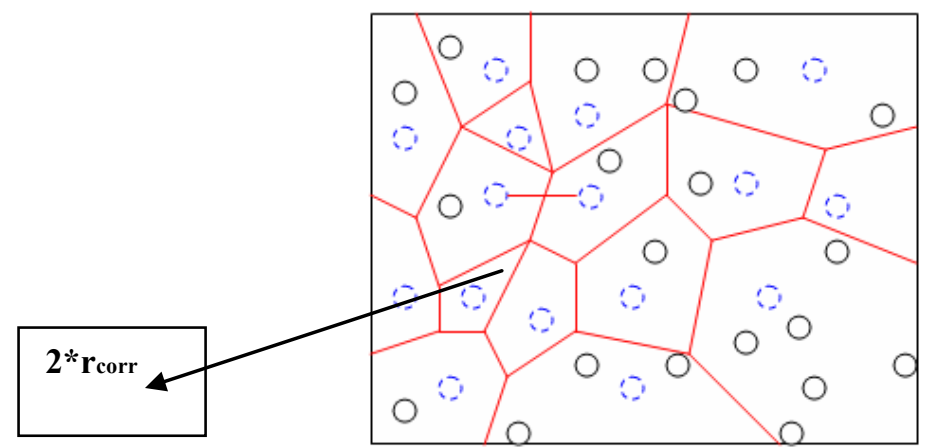

Fig.2.

Fig2 shows the representation of correlation region and Average distance between the selected representative node is $2 *$ rcorr. Correlation value is given by the expression,

$$
\operatorname{corr}\left\{S_{i}, S_{j}\right\}=\rho_{i, j}=K_{v}\left(d_{i, j}\right)=E\left[S_{i}, S_{j}\right] / \sigma^{2} s
$$

where $d_{i, j}=\rho_{s i}-\rho_{s j}$ where $\rho$ denotes the distance between nodes $n_{i}$ and $n_{j}$ located at coordinates $S_{i}$ and $S_{j}$, respectively, and $k v(\cdot)$ is the correlation model or covariance model. The covariance function is assumed to be non-negative and decrease monotonically with the distance.

\section{B. Pseudocode For The Proposed Model Is}

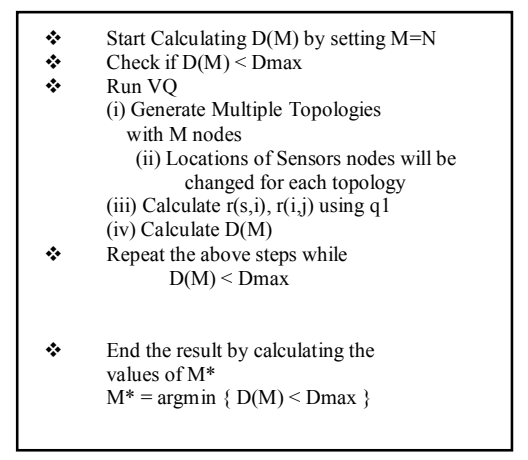

\section{Routing Protocol}

Two types of routing protocols are Proactive protocols and reactive protocols . Proactive Routing Protocols maintain fresh list of destinations and their routes by periodically distributing routing tables throughout the network. The main disadvantages are respective amount of data for maintenance and slow reaction on restructuring and failures. Reactive Protocols are also known as On-demand protocols. These type of protocol find a route on demand by flooding the network with route request packets. Two different routing protocols are considered to analyze spatial correlation. They are AODV and DSR. Dynamic Source Routing is shortly known as DSR and Adhoc On Demand Distance Vector Routing is known as AODV. DSR uses source routing and AODV uses a table driven routing framework with destination numbers. Timer activities are not directly involved in DSR but AODV takes into account of timer activities. AODV uses traditional routing tables, one entry per destination. Whereas in DSR, certain multiple route cache entries per destination are used. The main feature of DSR is source routing. i.e, the sender knows the complete hop-by-hop route to the destination. These routes are stored in a route cache. The data packets carry the source route in the packet header. Even through DSR and AODV share the on demand behaviour, their routing mechanisms are different. 
AODV is also appropriate for working in restrictive environments. It has the ability to intercommunicate with the endpoints which cannot be accessed directly.

\section{PERformance EVAluation}

In this section we present evaluated performance of the proposed model with DSR and AODV routing protocol, considering the graph between DSR and AODV protocol which is used in the implementation.

Fig. 3 provides the comparison made with respect to packet drop ratio. From the graph it is clear that the AODV protocol give better result by showing low packet drop ratio in comparison with DSR protocol.

Packet Drop Ratio $=($ Number of packets received at Base Station $) /($ Number of packets Transmitted by sink $)$

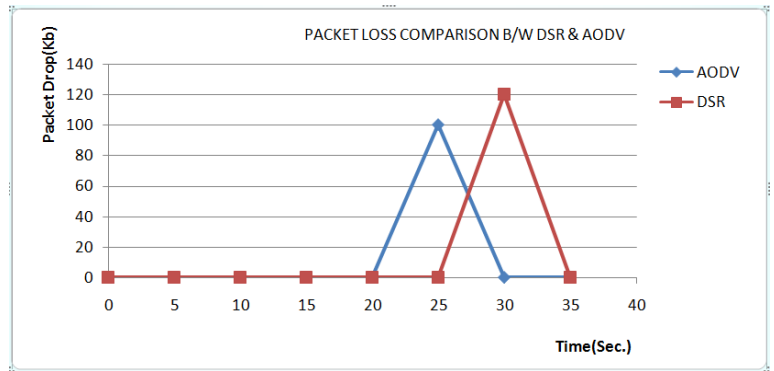

Fig3

(2) Fig.4 provides the comparison made with respect to energy consumption and it clear that by using AODV protocol the energy consumption can be reduced in comparison with DSR protocol.

Energy Consumption $=[($ Sum of energy expended by each node $) /($ Total number of data packets delivered $)]$

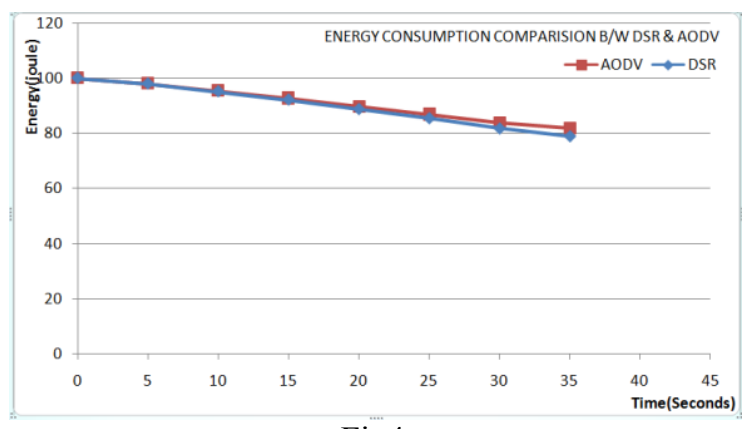

Fig4

\section{Conclusion}

A spatial correlation based energy efficient routing protocol with AODV and DSR routing protocol are used and the comparison made between the two routing protocol with respect to packet drop ratio and energy consumption. Iterative node selection algorithm and vector quantization methods are used to select a representative nodes in the sensor field. The comparison shows that by the use of spatial correlation and AODV routing protocol the performance is improved in comparison than the DSR routing protocol. In future the same process can be repeated for more nodes and corresponding modification can be done.

\section{Journal Papers:}

\section{REFERENCES}

[1] A.Rajeswari, P.T.Kalaivaani,, "Energy Efficient Routing Protocol for Wireless Sensor Networks Using Spatial Correlation Based Medium Access Control Protocol Compared with IEEE 802.11”, , 978-1-61284-764-1/11/\$26.00 C2011 IEEE

[2] Mehmet C. Vuran, Member, IEEE, and Ian F. Akyildiz, "Spatial Correlation-Based Collaborative Medium Access Control in Wireless Sensor Networks", IEEE/ACM TRANSACTIONS ON NETWORKING, VOL. 14, NO. 2, APRIL 2006

[3] Samir R. Das,Charles E. Perkins, Elizabeth M. Royer, Mahesh K. Marina," Performance Comparison of Two On-demand Routing Protocols for Ad Hoc Networks" IEEE INFOCOM 2000 conference

[4] David B. Johnson, David A. Maltz, Josh Broch, "DSR: The Dynamic Source Routing Protocol for Multi-Hop Wireless Ad Hoc Networks" http://www.monarch.cs.cmu.edu

[5] Charls E. Perkins,Elizabeth M. Royer"Ad-Hoc on demand Distence Vector Routing" google based search on "Ad-Hoc on demand Distence Vector Routing"

[6] Rajeev K. Shakya, Yatindra Nath Singh, and Nishchal K. Verma," A Novel Spatial Correlation Model for Wireless Sensor Network Applications", 978-1-4673-1989-8/12/\$31.00 C2012 IEEE

[7] The $n s$ Manual, http://www.isi.edu/nsnam/ns/ns-documentation.html

[8] The Network Simulator-ns-2. [Online]. Available:http://www.isi.edu/nsnam/ns/index.html 